\title{
Hospital outcomes of community-acquired COVID-19 versus influenza: Insights from the Swiss hospital-based surveillance of influenza and COVID-19
}

Georg Marcus Fröhlich ${ }^{1,2}$, Marlieke E. A. De Kraker ${ }^{3}$, Mohamed Abbas ${ }^{3}$, Olivia Keiser ${ }^{4}$, Amaury Thiabaud 4 , Maroussia Roelens ${ }^{4}$, Alexia Cusini ${ }^{5}$, Domenica Flury ${ }^{6}$, Peter W. Schreiber ${ }^{7}$, Michael Buettcher ${ }^{8}$, Natascia Corti ${ }^{9}$, Danielle Vuichard-Gysin ${ }^{10,11}$, Nicolas Troillet $^{11,12}$, Julien Sauser ${ }^{3}$, Roman Gaudenz ${ }^{13}$, Lauro Damonti ${ }^{14}$, Carlo Balmelli11,15, Anne Iten ${ }^{3}$, Andreas Widmer ${ }^{11,16}$, Stephan

Harbarth ${ }^{3,11}$, Rami Sommerstein ${ }^{11,14,17}$

1. HeartClinic Lucerne, Lucerne, Switzerland

2. Charité - Universitätsmedizin Berlin, Berlin, Germany

3. Infection Control program, Geneva University Hospitals and Faculty of Medicine, Geneva, Switzerland

4. Institute of Global Health of the University of Geneva, Geneva, Switzerland

5. Kantonsspital Graubünden, Department for General Medicine, Chur, Switzerland

6. Kantonsspital St. Gallen, St. Gallen, Switzerland

7. University Hospital Zurich, Division of Infectious Diseases and Hospital Epidemiology, and University of Zurich, Zurich, Switzerland

8. Cantonal Hospital Lucerne, Children's Hospital, Pediatric Infectious Diseases, Lucerne, Switzerland

9. Department for General Medicine, Klinik Hirslanden, Zürich, Switzerland

10. Department for General Medicine, Spital Thurgau, Frauenfeld, Switzerland

11. Swissnoso, the National Center for Infection Control, Bern, Switzerland

12. Department of Infectious Diseases, Central Institute, Valais Hospital, Sion, Switzerland

13. Department for General Medicine, Kantonsspital Nidwalden, Stans, Switzerland

14. Department of Infectious Diseases, Bern University Hospital, Bern, Switzerland

15. Ente Ospedaliero Cantonale Ticino, Division of Infection control and Hospital Epidemiology, Bellinzona, Switzerland

16. Department for Infectious Diseases, University Hospital Basel, Basel, Switzerland

17. Department of Health Sciences and Medicine, Clinic St. Anna, University of Lucerne, Lucerne, Switzerland

Correspondence: Rami Sommerstein (rami.sommerstein@hirslanden.ch)

Citation style for this article:

Fröhlich Georg Marcus, De Kraker Marlieke E. A., Abbas Mohamed, Keiser Olivia, Thiabaud Amaury, Roelens Maroussia, Cusini Alexia, Flury Domenica, Schreiber Peter W., Buettcher Michael, Corti Natascia, Vuichard-Gysin Danielle, Troillet Nicolas, Sauser Julien, Gaudenz Roman, Damonti Lauro, Balmelli Carlo, Iten Anne, Widmer Andreas, Harbarth Stephan, Sommerstein Rami. Hospital outcomes of community-acquired COVID-19 versus influenza: Insights from the Swiss hospitalWidmer Andreas, Harbarth Stephan, Sommerstein Rami. Hospital outcomes of community-acquired COVID-19 versus influenza: Insights from the
based surveillance of influenza and COVID-19. Euro Surveill. 2022;27(1):pii=2001848. https://doi.org/10.2807/1560-7917.ES.2022.27.1.2001848

Background: Since the onset of the COVID-19 pandemic, the disease has frequently been compared with seasonal influenza, but this comparison is based on little empirical data. Aim: This study compares in-hospital outcomes for patients with community-acquired COVID-19 and patients with community-acquired influenza in Switzerland. Methods: This retrospective multi-centre cohort study includes patients $>18$ years admitted for COVID-19 or influenza A/B infection determined by RT-PCR. Primary and secondary outcomes were in-hospital mortality and intensive care unit (ICU) admission for patients with COVID-19 or influenza. We used Cox regression (cause-specific and Fine-Gray subdistribution hazard models) to account for timedependency and competing events with inverse probability weighting to adjust for confounders. Results: In 2020, 2,843 patients with COVID-19 from 14 centres were included. Between 2018 and 2020, 1,381 patients with influenza from seven centres were included; 1,722 (61\%) of the patients with COVID-19 and $666(48 \%)$ of the patients with influenza were male $(p<0.001)$. The patients with COVID-19 were younger (median 67 years; interquartile range (IQR): $54-78$ ) than the patients with influenza (median 74 years; IQR: 61-84) $(p<0.001)$. A larger percentage of patients with COVID$19(12.8 \%)$ than patients with influenza (4.4\%) died in hospital ( $p<0.001)$. The final adjusted subdistribution hazard ratio for mortality was 3.01 (95\% Cl: 2.224.09; $p$ <0.001) for COVID-19 compared with influenza and 2.44 (95\% Cl: 2.00-3.00, p<0.001) for ICU admission. Conclusion: Community-acquired COVID-19 was associated with worse outcomes compared with community-acquired influenza, as the hazards of ICU admission and in-hospital death were about two-fold to three-fold higher.

\section{Introduction}

During the first wave, the clinical impact of the COVID19 pandemic was compared with seasonal influenza $[1,2]$. This comparison may have been because of its worldwide spread via respiratory particles and similar initial clinical presentation [3,4]. For both infections, the most common symptoms are cough, fever, dyspnoea or headache. However, COVID-19 appears more deleterious than influenza [5] as intensive care units (ICUs) were stressed, elective procedures were 
TABLE 1

Baseline characteristics of patients with COVID-19 (19 February - 22 July 2020) and influenza A/B (26 October 2018 - 26 March 2020) in the Swiss surveillance database, Switzerland $(\mathrm{n}=4,224)$

\begin{tabular}{|c|c|c|c|c|c|}
\hline Characteristics & \multicolumn{2}{|c|}{$\begin{array}{l}\text { SARS-CoV-2 } \\
(n=2,843)\end{array}$} & \multicolumn{2}{|c|}{$\begin{array}{c}\text { Influenza A/B } \\
(n=1,381)\end{array}$} & $\mathrm{p}$ value \\
\hline Age (years), median (IQR) & \multicolumn{2}{|c|}{$67(54-78)$} & \multicolumn{2}{|c|}{$74(61-84)$} & $<0.001$ \\
\hline Sex & $\mathrm{n}$ & $\%$ & $\mathrm{n}$ & $\%$ & \\
\hline Female & 1,121 & 39.4 & 715 & 51.8 & $<0.001$ \\
\hline \multirow{2}{*}{ Admission to university hospital } & $\mathrm{n}$ & $\%$ & $\mathrm{n}$ & $\%$ & \\
\hline & 1,597 & 56.2 & 935 & 67.7 & $<0.001$ \\
\hline BMI $\left(\mathrm{kg} / \mathrm{m}^{2}\right)$, median (IQR) & \multicolumn{2}{|c|}{$26.7(23.7,30.5)^{\mathrm{a}}$} & \multicolumn{2}{|c|}{$25.2(21.8,29.4)^{b}$} & $<0.001$ \\
\hline Comorbidities & $\mathrm{n}$ & $\%$ & $\mathrm{n}$ & $\%$ & \\
\hline Diabetes mellitus & 522 & $26.9^{c}$ & 291 & $28.2^{\mathrm{d}}$ & 0.578 \\
\hline Chronic cardiovascular disease & 737 & $38.0^{c}$ & 473 & $45.9^{d}$ & $<0.001$ \\
\hline Renal impairment & 374 & $19.3^{c}$ & 243 & $23.6^{\mathrm{d}}$ & 0.011 \\
\hline Chronic pulmonary disease & 413 & $21.3^{c}$ & 322 & $31.2^{\mathrm{d}}$ & $<0.001$ \\
\hline Chronic neurologic impairment & 276 & $14.2^{c}$ & 190 & $18.4^{\mathrm{d}}$ & 0.002 \\
\hline Haematological disorder & 39 & $2.0^{c}$ & 160 & $15.5^{\mathrm{d}}$ & $<0.001$ \\
\hline Chronic liver disease & 114 & $5.9^{c}$ & 57 & $5 \cdot 5^{\mathrm{d}}$ & 0.873 \\
\hline
\end{tabular}

BMI: body mass index; COVID-19: coronavirus disease; IQR: interquartile range; SARS-CoV-2: severe acute respiratory syndrome coronavirus 2.

a Missing values $\mathrm{n}(\%): 856(30 \%)$ as reporting of $\mathrm{BMI} /$ comorbidities was not mandatory and omitted by some centres.

${ }^{b}$ Missing values $\mathrm{n}(\%): 518(38 \%)$ as reporting of BMI/comorbidities was not mandatory and omitted by some centres.

c Missing values $\mathrm{n}(\%): 904$ (32\%) as reporting of BMI/comorbidities was not mandatory and omitted by some centres.

${ }^{\mathrm{d}}$ Missing values $\mathrm{n}(\%): 350$ (25\%) as reporting of BMI/comorbidities was not mandatory and omitted by some centres.

reduced, and national lockdowns resulted in considerable economic hardship for parts of the population.

During the influenza season in Switzerland, between 1,000 and 5,000 patients with influenza A/B need hospitalisation and up to 1,500 patients die each year [6]. In comparison, COVID-19 in Switzerland has already caused more than 704,000 infections, more than 29,000 hospitalisations, and more than 10,500 deaths as of 28 July $2021[7,8]$.

Although influenza is a well investigated disease with clear preventive and treatment strategies, including vaccination and anti-viral drugs, these strategies were not fully defined for COVID-19 at the time of writing. Several treatment strategies (e.g., dexamethasone and remdesivir) are already in use or are in the process of being approved [9-11]. However, vaccination appears to be the most efficacious preventive strategy with a conferred $95 \%$ protection against the wildtype virus [12].

So far, only a few retrospective studies have directly compared hospital outcomes of COVID-19 and influenza [13-16]. Two of these studies have demonstrated that comorbidities can affect the course of COVID-19 and influenza [13,14]. The comparison of COVID-19 and seasonal influenza is important from a public health perspective, as COVID-19 challenges the healthcare system in a different way. Therefore, we investigated the differences in mortality and ICU admission among patients hospitalised for COVID-19 and influenza in Switzerland.

\section{Methods}

Study population, data sources and variables This retrospective multi-centre cohort study included data from patients admitted to one of 14 hospitals in Switzerland, including all five Swiss university hospitals. Seven centres continuously collected data for all adult patients who were hospitalised for influenza $A / B$, including three university hospitals (start date: 28 October 2018). A total of 14 centres collected data for all adult patients who were hospitalised for COVID-19, including five overlapping centres with influenza and five university hospitals (start date: 19 February 2020).

All data were collected from the database Hospitalbased surveillance of COVID-19 and influenza cases in Switzerland. This database was originally initiated to collect data for all hospitalised influenza cases during the influenza season $2018 / 19$ by the Institute of Global Health at the University of Geneva and the Infection Control programme, Geneva University Hospital, Switzerland. A standardised, central REDCap-hosted electronic questionnaire for data collection was set up for data entry in the participating centres $[17,18]$.

Hospital data included patient demographics, main comorbidities, non-invasive or invasive ventilation, cardiovascular, pulmonary, or neurologic complications, 
Unadjusted crude outcomes for patients with microbiologically confirmed community-acquired COVID-19 (19 February 22 July 2020) or influenza A/B infections (26 October 2018 - 26 March 2020), Switzerland $(\mathrm{n}=4,224)$

\begin{tabular}{|c|c|c|c|c|c|}
\hline & \multicolumn{2}{|c|}{$\begin{array}{l}\text { SARS-CoV-2 } \\
(n=2,843)\end{array}$} & \multicolumn{2}{|c|}{$\begin{array}{c}\text { Influenza A/B } \\
(n=1,381)\end{array}$} & \multirow{2}{*}{$p$ value } \\
\hline & $\mathrm{n}$ & $\%$ & $\mathrm{n}$ & $\%$ & \\
\hline In-hospital deaths & 363 & 12.8 & 61 & 4.4 & $<0.001$ \\
\hline Admission to the ICU & 483 & 19.4 & 129 & 9.8 & $<0.001$ \\
\hline Invasive ventilation & 394 & 13.9 & 66 & 4.8 & $<0.001$ \\
\hline Respiratory complications & 2,082 & $93.6^{a}$ & 744 & $83.9^{b}$ & $<0.001$ \\
\hline Cardiac diseases & 556 & $25.0^{\mathrm{a}}$ & 212 & $23.9^{b}$ & 0.291 \\
\hline Neurologic impairment & 394 & $17.7^{\mathrm{a}}$ & 82 & $9.2^{\mathrm{b}}$ & $<0.001$ \\
\hline Renal impairment & 601 & $27.0^{\mathrm{a}}$ & 149 & $16.8^{\mathrm{b}}$ & $<0.001$ \\
\hline Length of hospital stay, median (IQR) & \multicolumn{2}{|c|}{$8(5-16)$} & \multicolumn{2}{|c|}{$7(4-13)$} & $<0.001$ \\
\hline Any antibiotic treatment & 1,539 & $61.7^{c}$ & 862 & $65.7^{\mathrm{d}}$ & $<0.001$ \\
\hline
\end{tabular}

COVID-19: coronavirus disease; ICU: intensive care unit; IQR: interquartile range; SARS-CoV-2: severe acute respiratory syndrome coronavirus 2.

a Missing values n (\%): 618 (22\%) as reporting of these complications was not mandatory and omitted by some centres.

${ }^{b}$ Missing values $\mathrm{n}(\%): 494$ (36\%) as reporting of these complications was not mandatory and omitted by some centres.

' Missing values n (\%): 349 (12) as reporting of these complications was not mandatory and omitted by some centres.

${ }^{\mathrm{d}}$ Missing values $\mathrm{n}(\%): 69$ (5) as reporting of these complications was not mandatory and omitted by some centres.

hospital and ICU admission and discharge date, as well as death $[17,18]$. Inclusion required a positive real-time polymerase chain reaction (RT-PCR) obtained from any respiratory sample.

Only data for completed hospitalisations of patients $>18$ years up to 22 July 2020 were included in the analyses. Potential hospital-acquired infections (with RT-PCR diagnosis obtained later than 2 days after admission) were excluded, as we wanted to focus on patients with influenza or COVID-19 present on admission. Sampling and RT-PCR processing were performed according to the local centre's protocols.

\section{Outcome measures}

The primary outcome measure was defined as inhospital all-cause mortality. Secondary outcome was admission to the ICU. Further exploratory end-points included cardiovascular, pulmonary, renal or neurologic complications during hospitalisation or length of stay and antibiotic treatment.

\section{Statistical analysis}

Pearson's chi-squared test was used for categorical data and Mann-Whitney $U$ test was used for ordinal data. Missing data were excluded for the calculation of the rate and the comparative tests.

For the primary and secondary outcome analyses, we used cause specific Cox hazard models to account for competing risks (hospital discharge for hospital mortality and discharge and death before ICU admission for ICU admission) to prevent overestimation of the impact of COVID-19 [19]. Patients who stayed more than 30 days were right-censored in all models. To adjust for differences in baseline characteristics, inverse probability weighting was applied using clinical and epidemiological factors present at admission significantly associated with influenza and COVID-19 as determined by univariate logistic regression models. We applied truncation at $1^{\text {st }} / 99^{\text {th }}$ percentile. In a second step, subdistribution hazard analysis using the Fine-Gray model was applied to determine the cumulative risk of death or ICU admission significantly associated with COVID19 or influenza [20]. The main analyses were adjusted for sex, age and university hospital as treatment centre (available for all cases).

A more detailed description of the methods and of additional analysis is provided in the Supplementary Materials. As the date of outcome was only recorded for mortality and ICU admission, we calculated the crude incidence for all other outcomes.

All analyses were performed in $R$, Version 4.02 ( $R$ Foundation, Vienna, Austria) using the packages survival, ipw, CausalGAM and cmprsk. Two-tailed tests were performed and $p$ values $<0.05$ were considered statistically significant.

\section{Ethical statement}

The study was approved by the Ethics Committee of the Canton of Geneva, Switzerland (CCER 2018-00577, 2020-00827). Data collection was also approved by all local ethics committees. The planning conduct and reporting of studies was in line with the Declaration of Helsinki, as revised in 2013. 


\section{FIGURE 1}

Cumulative incidence plot of mortality with discharge as competing risk by disease status, COVID-19 (19 February-22 July 2020) versus influenza (26 October 2018-26 March 2020), Switzerland $(\mathrm{n}=4,224)$

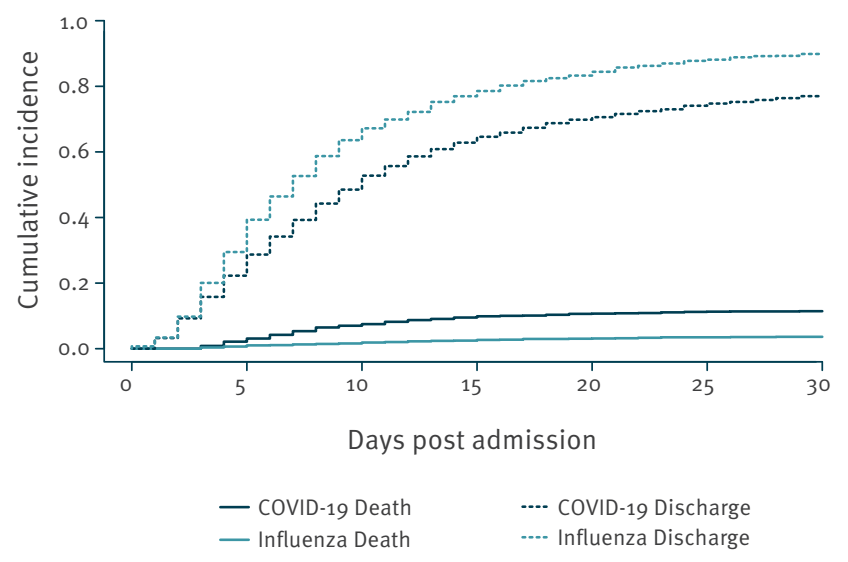

COVID-19: coronavirus disease.

\section{Results}

\section{Baseline characteristics}

In total, 4,441 patients were available from the two surveillance databases, of which 217 patients were excluded (170 for COVID-19 and 47 for influenza) because hospital admission or discharge date were missing.

This study includes 2,843 patients from 14 acute care hospitals in Switzerland with a confirmed diagnosis of community-acquired COVID-19 hospitalised between 19 February 2020 and 22 July 2020. This represents $50 \%$ of all reported hospitalisations in Switzerland during this period [21] $(4,569$ hospitalisations as of 3 September 2020). In addition, it includes 1,331 patients with community-acquired influenza $A(96.4 \%)$ and 50 patients (3.6\%) with community-acquired influenza B from seven centres in Switzerland hospitalised between 26 October 2018 and 26 March 2020. The majority of patients with COVID-19 (2,476/2,843, 87\%) as well as those with influenza A/B (1,220/1,381, 97\%) were directly referred to one of the participating hospitals. Several patients were transferred from local district hospitals to specialised facilities $(4.9 \%, n=139)$ of COVID-19 patients and 3.3\% $(n=46)$ of influenza patients). In addition, $4.8 \%(n=137)$ of the patients with COVID-19 and $5.2 \%(n=72)$ of the patients with influenza were transferred from a long-term care facility (LTCF) .

Patients with COVID-19 were younger (median 67 years; interquartile range (IQR): 54-78) than patients with influenza A/B (median 74 years; IQR: 61-84; $p<0.001$ ) and a greater percentage were male $(61 \%)$ than female $(48 \%)(p<0.001)$ (Table 1).

\section{Outcomes}

Table 2 provides an overview of the crude clinical outcomes for patients with COVID-19 and patients with influenza A/B. Crude hospital mortality among patients with COVID-19 was almost three times higher than for influenza A/B patients $(12.8 \%$ for patients with COVID-19 and $4.4 \%$ for patients with influenza A/B). ICU admission was also more frequent among patients with COVID-19 (19.4\%) compared with patients with influenza (9.8\%). Similarly, invasive ventilation was more common in patients with COVID-19 (13.9\%) compared to patients with influenza $(4.8 \%)$ ( $p<0.001)$. The crude overall length of hospital stay was 8 days for COVID (IQR: 5-16 days) and 7 days for influenza (IQR: 7-13) $(p<0.001)$.

The adjusted subdistribution hazard ratio (sdHR) for in-hospital mortality was 3.01 (95\% Cl: 2.22-4.09; $p<0.001$ ) for COVID-19 compared with influenza and 2.44 (95\% Cl: 2.00-3.00; $p<0.001$ ) for ICU admission (selected variables and balance of weights in Supplementary Table 1). The corresponding cumulative incidence plots for death and the competing risk discharge is shown in Figure 1 . The direct effect of COVID-19 on in-hospital death had a cause-specific hazard ratio (cSHR) of 2.58 (95\% Cl: $1.90-3.50$; $\mathrm{p}<0.001$ ), and the csHR for hospital discharge was 0.78 (95\% Cl: $0.72-0.84 ; \mathrm{p}<0.001$ ). That is, patients with COVID-19 stayed in hospital longer than influenza patients, which indirectly increased their risk of in-hospital death.

The largest increase in cumulative incidence of COVID19 deaths occurred from day 5 (2.1\%) to day 15 (9.9\%), whereas the increase in cumulative incidence of influenza deaths was $0.7 \%$ and $2.7 \%$, respectively. The cumulative incidence for influenza discharge was $29.4 \%$ on day 5 and $78.6 \%$ on day 15 , whereas the cumulative incidence for COVID-19 discharge on day 5 and day 15 was $22.2 \%$ and $64.6 \%$, respectively.

For the secondary outcome COVID-19-associated ICU admissions (Figure 2), the direct effect of COVID-19 had a csHR of 2.46 (95\% Cl: 2.00-3.01; p<001). Indirectly, COVID-19 altered the risk of ICU admission because patients with COVID-19 died earlier during their stay (csHR for death before ICU admission: 3.93; 95\% Cl: 2.61-5.95; $p<0.001)$. The csHR for the competing risk of being discharged before ICU admission was not significantly associated with COVID-19 compared to influenza (0.95; 95\% Cl: 0.87-1.03; $\mathrm{p}=0.181$ ).

Of note, the cumulative incidence of ICU admission, considering competing events, increased from $8.4 \%$ to $17.9 \%$ over the first 5 days for COVID-19, but it increased only from $7.0 \%$ to $9.2 \%$ for influenza over the same period. The results of the weighted csHR are summarised in Supplementary Table 3. 


\section{FIGURE 2}

Cumulative incidence plot of ICU admission with discharge and death before ICU admission as competing risk by disease status, COVID-19 (19 February-22 July 2020) versus influenza (26 October 2018-26 March 2020), Switzerland $(\mathrm{n}=4,224)$
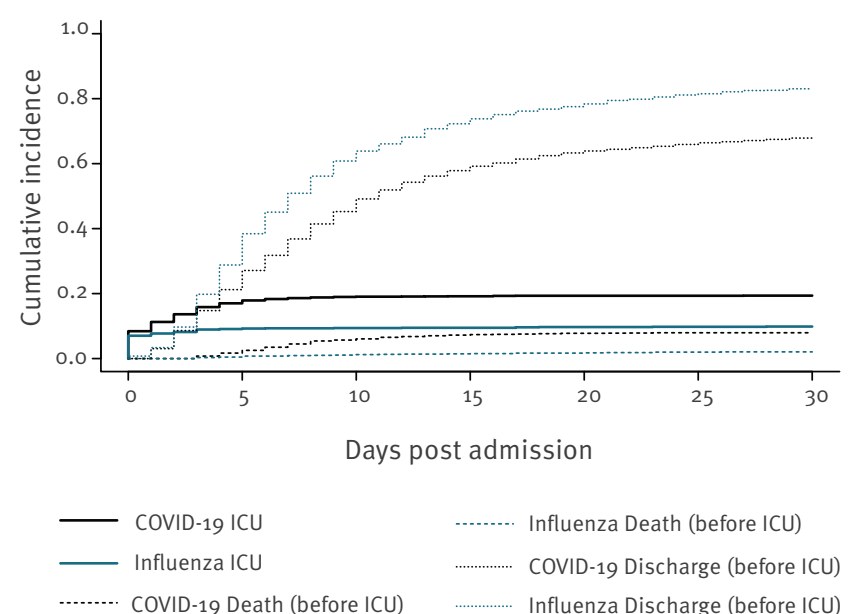

COVID-19: coronavirus disease; ICU: intensive care unit.

\section{Sensitivity analysis}

Of the 2,634 complete cases, the adjusted sdHR for mortality was slightly higher at 3.14 (95\% Cl: 2.11-4.68; $\mathrm{p}$ <0.001). The balance of weights for confounders are shown in Supplementary Table 2. The adjusted sdHR for mortality in the subgroup of 4,232 cases (excluding 209 direct transfers from LTCF) was 3.10 (95\% Cl: 2.23-4.32; $p<0.001)$, and the adjusted sdHR for mortality in the subgroup of 4,391 Influenza A and COVID-19 cases (excluding 50 with Influenza B) was 2.87 (95\% $\mathrm{Cl}$ : 2.11-3.90; $\mathrm{p}$ <0.001). Finally, the adjusted sdHR for ICU admission in 4,139 cases, excluding the 302 patients directly admitted to the ICU on the day of hospitalisation, was higher at 5.05 (95\% Cl: 3.54-7.20; $\mathrm{p}$ <0.001). The cumulative incidence plot is shown in Supplementary Figure 1.

\section{Discussion}

This study directly compares the outcomes of patients hospitalised for influenza A/B or COVID-19 in a nationwide Swiss surveillance database. After considering competing events as well as imbalances between patient groups, we found that community-acquired COVID-19 was associated with a threefold increase in the cumulative risk of in-hospital death compared with influenza A/B. This increase was associated with a higher daily mortality risk as well as an increased length of hospital stay for patients with COVID-19. Similarly, patients with COVID-19 had a more than twofold increased risk of being transferred to the ICU than influenza patients.

These numbers are in line with a large French study by Piroth et al., where the in-hospital mortality rate was
16.9\% for COVID-19 and 5.8\% for influenza. Similarly, they reported that the need for ICU admission was higher in patients with COVID-19 (16.3\%) than in patients with influenza (10.8\%) [15].

The COVID-19 pandemic poses a tremendous medical and socioeconomic burden for many countries. Early in the pandemic, however, COVID-19 was downplayed in some communications as only a 'little flu' $[1,22,23]$. Now we know that the characteristics as well as the course of COVID-19 appear to be more serious than for influenza in several aspects. Severe acute respiratory syndrome coronavirus 2 (SARS-CoV-2) and in particular several of its variants appear to be more contagious than the influenza virus, with a reproductive number of $2-2.5$, and contagious even in patients without symptoms or before symptoms appear [3]. Moreover, according to the World Health Organization (WHO), in unvaccinated populations $15 \%$ of COVID-19 cases might need hospitalisation and $5 \%$ of hospitalised patients require ventilation [3]. In comparison, a study from Germany of 34,493 patients with influenza found that $3.7 \%$ of these patients needed hospitalisation, and $5 \%$ of these received invasive ventilation [24]. In our study, $13.9 \%$ of the hospitalised patients with COVID-19 needed mechanical ventilation, compared to $4.7 \%$ of the hospitalised patients with influenza. These numbers underline the clinical importance of COVID-19.

In our study, patients with COVID-19 were predominantly male with a median age of 67 years, and patients with influenza were predominantly female with a median age of 74 years. Therefore, the years of potential life lost are likely to be higher in COVID-19. This hypothesis is supported by the finding that the patients with COVID-19 were less likely to have some of the comorbidities than patients with influenza. However, all the clinical outcomes for patients with COVID-19 were worse than those for patients with influenza and persisted even after adjustment for possible confounders. The WHO estimates case fatality rates of $3-4 \%$ for COVID-19, whereas the infection fatality rates for seasonal influenza are usually much lower, around $0.1 \%$ [2]. In our Swiss hospital surveillance, the cumulative incidence of mortality of hospitalised patients with COVID-19 up to day 30 was $11.4 \%$ compared with $3.6 \%$ in hospitalised influenza patients. Previous studies reported mortality rates of hospitalised patients with COVID-19 between $4 \%$ and $28 \%[25,26]$.

In the presented Swiss cohort, the cumulative incidence of patients with COVID-19 with ICU admission up to day 30 was $19.4 \%$ compared with $9.9 \%$ among influenza patients. Interestingly, patients with influenza who needed intensive care were transferred to ICU soon after hospital admission. In contrast, the proportion of ICU transfers for patients with COVID-19 increased steadily up to 5 days after hospital admission.

Apart from respiratory complications, neurologic impairment or kidney failure were also more common 
among the COVID-19 patients. It is unclear whether this finding might be explained by vasculitis and thromboembolic events, which are common in COVID-19 [27]. Surprisingly, there was no difference in cardiovascular complications. Although antibiotic therapy was more common in patients with influenza in this Swiss cohort, it remains unclear whether influenza is associated with pulmonary bacterial superinfection more often than COVID-19.

The present study has several limitations. Influenza seasons may vary considerably in their severity depending on circulating viruses. In the presented analysis, only data from two seasons - 2018/19 $(n=1,010)$ and 2019/20 ( $n=371)$ - were available. In addition, detection bias of in-hospital influenza cases cannot fully be excluded. In this regard, a difference was noted between university hospitals and non-university hospitals: detection of influenza cases was statistically significant more frequently in university hospitals. This may hint at a need for more stringent case detection for COVID-19, especially in non-university hospitals. Therefore, some mild influenza cases may have been missed and this may have even led to an underestimation of the severity of COVID-19. Nevertheless, the main analysis was adjusted for type of hospital (university and other). Irrespective of hospital type, the results remained unchanged. Another possible bias is related to missing data. For about one-third of the patients in the database, comorbidity data were lacking because it was not mandatory for the surveillance. As missingness was centre-specific (missing at random) and only related to independent variables, we conducted complete case analyses for the primary outcome. The point-estimate of the analysis including all patients but excluding BMI and comorbidities from the propensity weights fell within the $95 \% \mathrm{Cl}$ of the main analysis, indicating that excluding incomplete cases did not introduce a major bias.

The impact of various treatment strategies could not be analysed in the presented Swiss cohort at the time of writing. It can be assumed that mortality for the patients with COVID-19 was relatively high, as the majority of the presented cases occurred early during the COVID-19 pandemic and knowledge about the best supportive treatment strategies has slowly increased. As such, the current findings may not apply to future COVID-19 cases.

Data on type of COVID or influenza strains were not available, but influenza $B$ cases were rare (only 50 patients), a finding that suggests that there was no substantial bias for grouping influenza A and B together.

Finally, since no data were available for co-infection of influenza virus and SARS-CoV-2, this was not considered. However, several case reports found that only $4 \%$ of patients with COVID-19 had a co-infection with influenza virus [28], and no difference in the course of disease was noted in comparison to SARS-CoV-2 infections alone $[28,29]$.

\section{Conclusion}

In this retrospective Swiss multi-centre hospital cohort study, COVID-19 was associated with a three-fold higher hazard for hospital mortality and a two-fold higher risk of ICU transfer compared with seasonal influenza A/B infection. These results have important implications for decisions about public health interventions in the current COVID-19 pandemic.

\section{Acknowledgements}

We thank all participating centres for providing their surveillance data.

These data were collected in collaboration with the Swiss Federal Office of Public Health, with contributions from the Clinical Research Centre, Geneva University Hospitals and Faculty of Medicine, Geneva. The authors want to especially thank Markus Schneemann, Department for General Medicine, Spitäler Schaffhausen, Geissbergstrasse 81, 8208 Schaffhausen, Switzerland, Laurence Senn, Centre Hospitalier Universitaire Vaudoise, Lausanne, Lisa Brockhaus, Department for General Medicine, Hôpital du Jura, Delémont, and Jonas Marschall, Bern University Hospital for acting as local principal investigators in the hospital-based surveillance.

\section{Funding statement}

Swiss Hospital Surveillance for Influenza and COVID19 was supported by the Swiss Federal Office of Public Health.

\section{Data sharing statement}

Swiss COVID-19/Influenza Hospital Surveillance data access is available at https://www.unige.ch/medecine/hospital-covid/files/4015/9427/6937/CH_SUR_ Multicentric_process_final.pdf

\section{Conflict of interest}

Marlieke E.A. de Kraker was supported by the IMI Joint Undertaking (JU) (grant 115523), Combatting Bacterial Resistance in Europe, with resources including financial contribution from the EU's Seventh Framework Programme and in-kind contributions from companies in the European Federation of Pharmaceutical Industries and Associations (EFPIA). Peter W Schreiber received grant support from the career funding programme Filling the Gap of the Medical Faculty of the University of Zurich.

\section{Authors' contributions}

Conception and design: GMF, RS, MEAdK, SH, AW, CB, MA.

Development of the surveillance system: AI, OK, AT, MR.

Acquisition of data: AC, DF, PWS, MB, NC, DVG, NT, RG, LD, CB, AW.

Statistics: MEAdK, RS, SH, JS, MA.

Writing of first draft: GF, RS, MEAdK. 
Interpretation and analysis: All authors.

RS is guarantor of the paper. All authors have seen, critically revised and approved the manuscript.

\section{References}

1. Molina B. Facebook pulls Trump post comparing COVID-19 to flu; Twitter adds warning. Geneva: USA Today. 6 Oct 2020. Available from: https://eu.usatoday.com/story/ tech/2020/10/06/trump-post-covid-19-draws-removalfacebook-gets-twitter-warning/3637613001/

2. Beer T. All The Times Trump Compared Covid-19 To The Flu, Even After He Knew Covid-19 Was Far More Deadly. Jersey. Forbes. 2020;10. Available from: https://www.forbes.com/ sites/tommybeer/2020/09/10/all-the-times-trump-comparedcovid-19-to-the-flu-even-after-he-knew-covid-19-was-far-moredeadly/?sh $=35168 \mathrm{c} 77$ fgd 2

3. World Health Organization (WHO). Q\&As on COVID-19 and related health topics. Coronavirus disease (COVID-19): similarities and differences between COVID-19 and Influenza. Geneva: WHO; 30 Sep 2020. Available from: https://www.who. int/emergencies/diseases/novel-coronavirus-2019/questionand-answers-hub/coronavirus-disease-covid-19-similaritiesand-differences-with-influenza

4. Petersen E, Koopmans M, Go U, Hamer DH, Petrosillo N, Castelli F, et al. Comparing SARS-CoV-2 with SARS-CoV and influenza pandemics. Lancet Infect Dis. 2020;20(9):e23844. https://doi.org/10.1016/S1473-3099(20)30484-9 PMID 32628905

5. The World Bank. The Global Economic Outlook During the COVID-19 Pandemic: A Changed World. Washington: The World Bank; 8 Jun 2020. Available from: https://www.worldbank.org/ en/news/feature/2020/06/o8/the-global-economic-outlookduring-the-covid-19-pandemic-a-changed-world

6. Bundesamt für Gesundheit BAG. Saisonale Grippe 2011/2012: Zeit, sich impfen zu lassen. [Seasonal influenza 2011/2012: Time to get vaccinated]. BAG Bulletin. 2011;42:899901. German. Available from: https://www.infovac.ch/ de/?option=com_gd\&view=listing\&fid=706\&task=ofile

7. John Hopkins University. Coronavirus map. Baltimore: Johns Hopkins University. [Accessed: 28 Jul 2021]. Available from: https://coronavirus.jhu.edu/map.html

8. Bundesamt für Gesundheit BAG. Dashboard. Bern: Bundesamt für Gesundheit BAG. 2021. German. Available from: https:// www.covid19.admin.ch/de/overview

9. Grein J, Ohmagari N, Shin D, Diaz G, Asperges E, Castagna A, et al. Compassionate Use of Remdesivir for Patients with Severe Covid-19. N Engl J Med. 2020;382(24):2327-36. https:// doi.org/10.1056/NEJMoa2007016 PMID: 32275812

10. Group RC, Horby P, Lim WS, Emberson JR, Mafham M, Bell JL, et al. Dexamethasone in Hospitalized Patients with Covid-19 Preliminary Report. N Engl J Med. 2020.

11. Elekhnawy E, Kamar AA, Sonbol F. Present and future treatment strategies for coronavirus disease 2019. Futur J Pharm Sci. 2021;7(1):84. PMID: 33850936

12. Polack FP, Thomas SJ, Kitchin N, Absalon J, Gurtman A, Lockhart S, et al. Safety and Efficacy of the BNT162 b2 mRNA Covid-19 Vaccine. N Engl J Med. 2020;383(27):2603-15. https:// doi.org/10.1056/NEJMoa2034577 PMID: 33301246

13. Faury H, Courboules C, Payen M, Jary A, Hausfater P, Luyt C, et al. Medical features of COVID-19 and influenza infection: A comparative study in Paris, France. J Infect. 2020. PMID: 32798533

14. Zayet S, Kadiane-Oussou NJ, Lepiller Q, Zahra H, Royer PY, Toko L, et al. Clinical features of COVID-19 and influenza: a comparative study on Nord Franche-Comte cluster. Microbes Infect. 2020;22(9):481-8. https://doi.org/10.1016/j. micinf.2020.05.016 PMID: 32561409

15. Piroth L, Cottenet J, Mariet AS, Bonniaud P, Blot M, TubertBitter P, et al. Comparison of the characteristics, morbidity, and mortality of COVID-19 and seasonal influenza: a nationwide, population-based retrospective cohort study. Lancet Respir Med. 2021;9(3):251-9. https://doi.org/10.1016/ S2213-2600(20)30527-0 PMID: 33341155

16. Xie Y, Bowe B, Maddukuri G, Al-Aly Z. Comparative evaluation of clinical manifestations and risk of death in patients admitted to hospital with covid-19 and seasonal influenza: cohort study. BMJ. 2020;371:m4677. https://doi.org/10.1136/ bmj.m4677 PMID: 33323357

17. University of Geneva. Hospital based surveillance of influenza in Switzerland - a pilot study. Geneva: University of Geneva. [Accessed: 24 Dec 2021]. Available from: https://www.unige. ch/medecine/hospital-flu/
18. University of Geneva. Hospital based surveillance of Covid-19 in Switzerland. Geneva: University of Geneva. [Accessed: 24 Dec 2021]. Available from: https://www.unige.ch/medecine/ hospital-covid/

19. Lau B, Cole SR, Gange SJ. Competing risk regression models for epidemiologic data. Am J Epidemiol. 2009;170(2):244-56. https://doi.org/10.1093/aje/kwp107 PMID: 19494242

20. Fine IP, Gray RJ. A Proportional Hazards Model for the Subdistribution of a Competing Risk. J Am Stat Assoc. 1999;94(446):496-509. https://doi.org/10.1080/01621459.199 9.10474144

21. Statista. Number of hospitalizations due to the coronavirus (COVID-19) in Switzerland in 2020. New York: Statista. [Accessed: 24 Dec 2021]. Available from: https://www.statista. com/statistics/1110045/coronavirus-covid-19-hospitalizationsnumber-age-group-switzerland/

22. Kerr S, Felling M, Jordan D. Is It Like the Flu? Is It a Major Threat? Trump's Changing Coronavirus Message. New York: New York Times. 3 Apr 2020. Available from: https://www. nytimes.com/video/us/politics/100000007067717/trumpscoronavirus-statements.html

23. Walsh NP, Shelley J, Duwe E, Bonnett W. Bolsonaro calls coronavirus a 'little flu'. Atlanta: CNN; 25 May 2020. Available from: https://edition.cnn.com/2020/05/23/americas/brazilcoronavirus-hospitals-intl/index.html

24. von der Beck D, Seeger W, Herold S, Günther A, Löh B. Characteristics and outcomes of a cohort hospitalized for pandemic and seasonal influenza in Germany based on nationwide inpatient data. PLoS One. 2017;12(7):e0180920. https://doi.org/10.1371/journal.pone.0180920 PMID: 28708896

25. Wang D, Hu B, Hu C, Zhu F, Liu X, Zhang J, et al. Clinical Characteristics of 138 Hospitalized Patients With 2019 Novel Coronavirus-Infected Pneumonia in Wuhan, China. JAMA. 2020;323(11):1061-9. https://doi.org/10.1001/jama.2020.1585 PMID: 32031570

26. Zhou F, Yu T, Du R, Fan G, Liu Y, Liu Z, et al. Clinical course and risk factors for mortality of adult inpatients with COVID-19 in Wuhan, China: a retrospective cohort study. Lancet. 2020;395(10229):1054-62. https://doi.org/10.1016/S01406736(20)30566-3 PMID: 32171076

27. Mondal S, Quintili AL, Karamchandani K, Bose S. Thromboembolic disease in COVID-19 patients: A brief narrative review. I Intensive Care. 2020;8(1):70. https://doi. org/10.1186/s40560-020-00483-y PMID: 32939266

28. Ding Q, Lu P, Fan Y, Xia Y, Liu M. The clinical characteristics of pneumonia patients coinfected with 2019 novel coronavirus and influenza virus in Wuhan, China. J Med Virol. 2020;92(9):1549-55. https://doi.org/10.1002/jmv.25781 PMID: 32196707

29. Cuadrado-Payán E, Montagud-Marrahi E, Torres-Elorza M, Bodro M, Blasco M, Poch E, et al. SARS-CoV-2 and influenza virus co-infection. Lancet. 2020;395(10236):e84. https://doi. org/10.1016/S0140-6736(20)31052-7 PMID: 32423586

\section{License, supplementary material and copyright}

This is an open-access article distributed under the terms of the Creative Commons Attribution (CC BY 4.0) Licence. You may share and adapt the material, but must give appropriate credit to the source, provide a link to the licence and indicate if changes were made.

Any supplementary material referenced in the article can be found in the online version.

This article is copyright of the authors or their affiliated institutions, 2022. 